\title{
Achieving Fine Bit-Rate Granularity with Hybrid Subcarrier Modulation
}

\author{
Fernando Guiomar and Andrea Carena \\ Dip. di Elettronica e Telecomunicazioni, Politecnico di Torino, Corso Duca degli Abruzzi, 24, 10129 Torino, Italy \\ fernando.guiomar@polito.it
}

\begin{abstract}
We assess the performance of hybrid subcarrier modulation to enable timeindependent fine bit-rate granularity. Employing polarization interleaving and optimized format allocation, we found similar performance to time-domain hybrid formats.
\end{abstract}

OCIS codes: (060.2360) Fiber optics links and subsystems; (060.1660) Coherent communications.

\section{Introduction}

Standard m-QAM modulation associated with fixed symbol-rate transceivers provides limited bit-rate granularity to transmission systems. Indeed, the most commonly used square polarization multiplexed QAM constellations (PMQPSK, PM-16QAM and PM-64QAM) in optical communication systems imply a minimum step of 4 bits per symbol between them, thus limiting the network flexibility. In order to provide continuous bit-rate granularity while maintaining a fixed symbol-rate transceiver, time-domain hybrid modulation formats (TDHMF) have been recently proposed and assessed both numerically and experimentally [1-3]. TDHMF introduces time-dependent modulation, in which low- and high-cardinality formats are mixed to yield any desirable transmission bit-rate. If properly designed, the nonlinear propagation penalties associated with TDHMF were shown to be almost fully suppressed, enabling to perform similarly to the Gaussian noise (GN) model predictions [3]. The main disadvantage of this flexible solution then lies on the extra digital signal processing (DSP) complexity that may be required to process and equalize a time-dependent modulation format.

Recently it has been shown that symbol-rate optimization (SRO) can be applied to reduce non-linear propagation impact $[4,5]$. Because optimal symbol-rates for standard systems result to be very low, in the order of few GBaud, subcarrier multiplexing (SCM) has been proposed as a solution to get all advantages of SRO without increasing the transceiver count and consequently the cost and complexity of system implementation. For this reason SCM has been attracting an increasing attention as a high-performing alternative for long-haul optical systems. Besides the SRO benefits, SCM can also allow an increased flexibility relatively to single-carrier systems. A recent experimental work has exploited the SCM concept to design high bit-rate optical channels that are more robust to tight optical filtering, by using PM-QPSK in edge subcarriers and PM-16QAM in the central ones [6].

In this work, we propose the use of frequency domain hybrid modulation formats (FDHMF) (hybrid subcarrier modulation) as a time-invariant alternative to TDHMF. We numerically assess its performance and flexibility in standard single-mode fiber (SSMF) links with net bit-rate per channel ranging from 200G (pure PM-16QAM) to 300G (pure PM-64QAM).

\section{Results}

The simulation scenario is as follows. We consider 15 WDM channels with an inter-channel spacing of $37.5 \mathrm{GHz}$. Each channel runs at an aggregate symbol rate of 32 GBaud and can be either a single-carrier or a SCM solution. In all cases square root raised-cosine spectral shaping with $\rho=0.05$ roll-off is applied to each carrier. For all SCM cases the subcarrier spacing is set to $\Delta f=(1+\rho) \cdot R_{S C}$, where $R_{S C}$ is the subcarrier symbol rate. We also consider TDHMF as a reference: the power-ratio between formats in both FDHMF and TDHMF is set in order to force the same BER performance [3] at a target BER of $10^{-2}$, yielding a power-ratio of $\sim 5.83 \mathrm{~dB}$ between PM-16QAM and PM-64QAM tributaries. The signal is then propagated over a periodical link made of standard single-mode fiber (SSMF) $\left(\alpha=0.2 \mathrm{~dB} / \mathrm{km}, \beta_{2}=-21.4 \mathrm{ps}^{2} / \mathrm{km}\right.$ and $\left.\gamma=1.3 \mathrm{~W}^{-1} \mathrm{~km}^{-1}\right)$ with a full-bandwidth split-step algorithm. After each fiber span, EDFA-based lumped optical amplification is applied with $5 \mathrm{~dB}$ noise figure. At the receiver we consider a standard polarization diversity opto-electronic front-end. After ADC sampling at 2 samples per symbol (considering the aggregate symbol rate of 32 GBaud), we first compensate for chromatic dispersion with a static frequency-domain equalizer. Then, in the case of FDHMF, each subcarrier is digitally filtered and down-converted to 


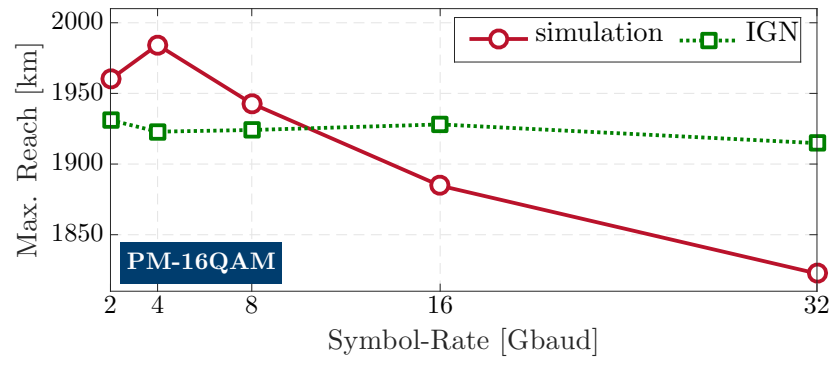

(a)

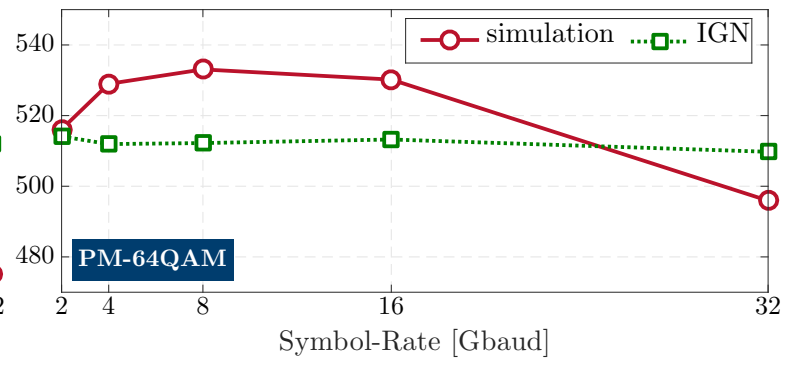

(b)

Fig. 1: Impact of symbol-rate optimization using a) PM-16QAM and b) PM-64QAM modulation formats.

baseband. A data-aided least-mean squares (DA-LMS) filter is employed for the adaptive linear equalization of both TDHMF and FDHMF. Finally, after symbol decoding the BER is determined by direct error counting.

Fig. 1 shows the SRO simulation results obtained for pure PM-16QAM (200G) and pure PM-64QAM (300G), starting from a reference symbol-rate of 32 GBaud (single-carrier) and gradually reducing it by factors of 2 down to 2 GBaud (16 subcarriers). Even though it is not able to predict symbol-rate optimization, the incoherent GN (IGN) model is used here as a reference performance curve. Taking into account the obtained optimal symbol-rates (4 GBaud for PM-16QAM and 8 GBaud for PM-64QAM), we have defined a FDHMF configuration composed of 8 subcarriers, enabling a net bit-rate granularity of up to $12.5 \mathrm{~Gb} / \mathrm{s}$.

Using such a 8-subcarrier scenario, a large number of possible FDHMF configurations is possible by applying different permutations of modulation format assignment to each subcarrier. To simplify our approach, we have defined 3 possible configurations: i) frequency interleaving of the low- and high-cardinality formats; ii) allocating the highcardinality format to the edge subcarriers and the low-cardinality format to the center subcarriers; and iii) applying the reverse of ii). The obtained simulation results in terms of maximum reach versus launched power is shown in Fig. 2 for $225 \mathrm{G}, 250 \mathrm{G}$ and $275 \mathrm{G}$ net bit-rates. Note that, although the selected 8-subcarrier FDHMF configuration allows to adjust the bit-rate in steps of $12.5 \mathrm{~Gb} / \mathrm{s}$, in this paper we will restrict our analysis to a $25 \mathrm{~Gb} / \mathrm{s}$ granularity.

The maximum reach is calculated taking into account the average BER among all subcarriers. In order to assess the performance of each modulation format, we also plot the average BER of each set of subcarriers associated with PM-16QAM and PM-64QAM modulation formats. In all simulated cases, the two modulation formats are shown to perform very similarly in the linear regime, proving that the same BER condition is actually correctly accomplished. However, even for mildly nonlinear propagation, the performance of the two sets of subcarriers associated with each modulation format start to detach from each other. Due to the higher launched power per subcarrier, there is a clear penalty associated with the PM-64QAM subcarriers, which tends to become more pronounced as we reduce the number of PM-64QAM tributaries. Indeed, there is approximately a $2 \mathrm{~dB}$ gap between the maximum reach of PM-16QAM and PM-64QAM for the 225G case, whereas the gap reduces to about $1 \mathrm{~dB}$ for the 275G. Although the reasoning for this behavior still requires further investigation, a possible justification is the offset between the subcarrier power and the average channel power. While for $225 \mathrm{G}$ case the PM-64QAM subcarriers are modulated at $\sim 3.5 \mathrm{~dB}$ above the average channel power, for the $275 \mathrm{G}$ case this gap is reduced to less than $0.9 \mathrm{~dB}$. Frequency-interleaving is also shown to be the best approach both for the $250 \mathrm{G}$ and $275 \mathrm{G}$ cases, as it reduces the nonlinear interference between the high-power PM-64QAM subcarriers. In contrast, slightly higher performance can be achieved in the 225G case by assigning PM-64QAM to the two edge subcarriers, since nonlinear interference is lower there, owing to the $\sim 4 \mathrm{GHz}$ inter-channel gap in the $37.5 \mathrm{GHz}$ grid.

Employing the best performing subcarrier allocation options, Fig. 3 summarizes the obtained maximum reach versus net bit-rate considering a) the worst performing subcarrier, b) the best performing subcarrier and c) the weighted average BER of all subcarriers (taking into account the different number of bits per symbol assigned to each modulation format). Again, the IGN model is used as a performance reference, and TDHMF results are also shown considering a single-carrier modulated at 32 GBaud and a frame-size of 4 symbols. As it has been shown to be effective on mitigating the nonlinear penalties associated with TDHMF, polarization interleaving (PI) is also applied here for both FDHMF and TDHMF. From Figs. 3a and $3 \mathrm{~b}$ we observe that PI tends to enable important benefits on the worst performing FDHMF carriers (64QAM) both at $225 \mathrm{G}$ and $250 \mathrm{G}$ and a sizeable penalty on the best performing carrier (16QAM) at $250 \mathrm{G}$ and $275 \mathrm{G}$. Note that for $225 \mathrm{G}(6 \times 16 \mathrm{QAM}+2 \times 64 \mathrm{QAM})$ the PI benefit comes from the reduction of optical power on the 64QAM edge carriers, thus reducing their nonlinear interference.This PI benefit on the worst subcarrier is also obtained for the $250 \mathrm{G}$ case $(4 \times 16 \mathrm{QAM}+4 \times 64 \mathrm{QAM})$, but now associated with a similar penalty on the best 


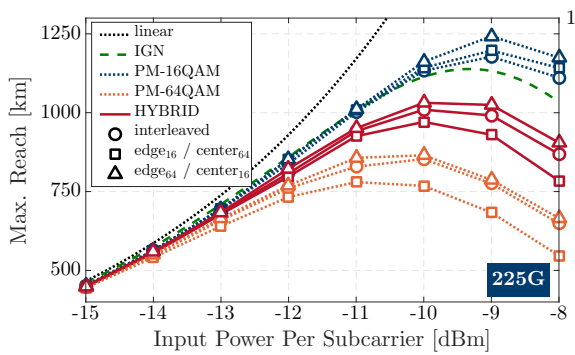

(a)

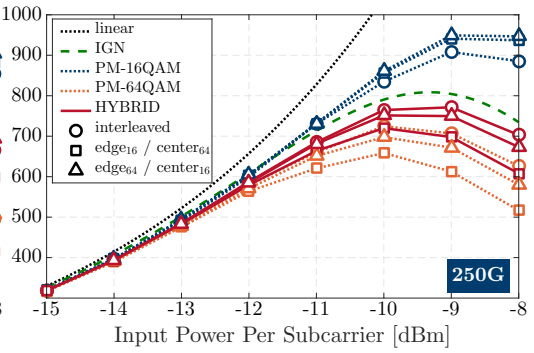

(b)

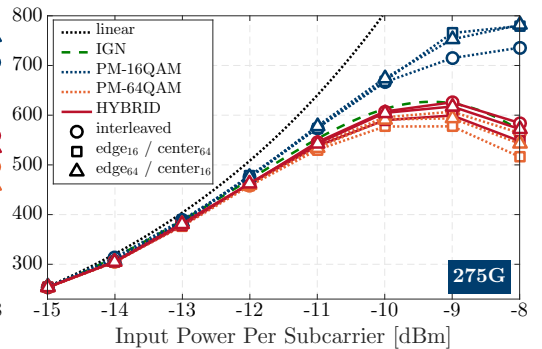

(c)

Fig. 2: Maximum reach versus the input power per subcarrier. a) $225 \mathrm{G}$, b) $250 \mathrm{G}$ and c) $275 \mathrm{G}$. Dashed green curves show the max-reach estimation using the IGN model for the required average OSNR.

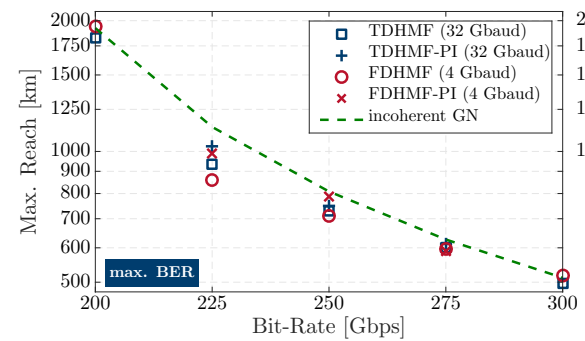

(a)

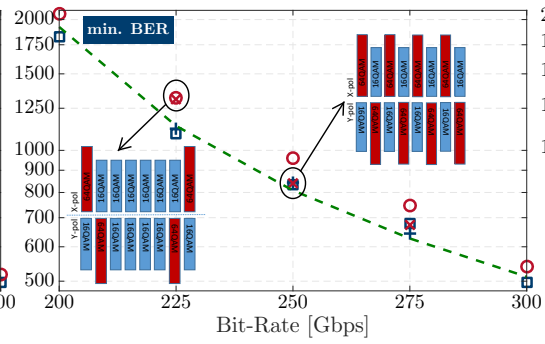

(b)

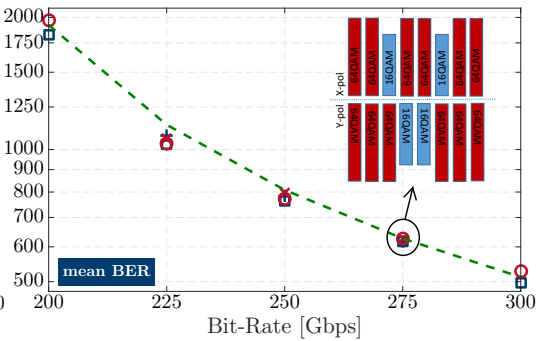

(c)

Fig. 3: Maximum reach versus net bit-rate employing FDHMF at 4 GBaud (8 subcarriers) and TDHMF at 32 GBaud (4 symbols per frame). Maximum reach is calculated based on a) the worst performing subcarrier, b) the best performing subcarrier and c) the average performance. The insets represent the selected allocation of modulation formats to each subcarrier.

performing subcarriers (16QAM), which now suffer from stronger intra-subcarrier nonlinear interference due to the insertion of a higher power polarization tributary. As a result, the two phenomena tend to cancel out, yielding negligible PI impact on the average system performance (see Fig. 3c). In turn, for the $275 \mathrm{G}$ case the small PI benefit over the 64QAM subcarriers tends to cancel with the penalty created on the 16QAM subcarriers, again yielding negligible PI impact in terms of the average BER of the channel. In summary, although the impact on the average BER performance is negligible, PI is shown to significantly reduce the gap between the best and worst performing subcarriers, which may be an important benefit in terms of the complexity and effectiveness of forward-error correction (FEC). Finally, Fig. $3 c$ shows that FDHMF and TDHMF perform similarly in terms of average BER for all considered net bit-rates.

\section{Conclusions}

By optimizing the modulation format allocation, hybrid subcarrier modulation is shown to be an interesting alternative to TDHMF, providing similar performance and enabling time-independent processing in the transceiver. Due to the impact of nonlinearities caused by the power unbalance between subcarriers, a strong performance gap was found between different modulation formats, which can be partially mitigated through polarization interleaving.

\section{Acknowledgements}

This work was partially supported by the European Commission through a Marie Skodowska-Curie individual fellowship, project Flex-ON (653412). We also acknowledge Synopsys Inc. for supplying the OptSim simulator in the framework of a research contract.

\section{References}

[1] W.-R. Peng et al, "Hybrid QAM transmission techniques for single-carrier ultra-dense WDM systems," in 16th Opto-Electronics and Communications Conference (OECC), 2011, pp. 824825.

[2] Q. Zhuge et al, "Spectral efficiency-adaptive optical transmission using time domain hybrid QAM for agile optical networks," J. Lightw. Technol., vol. 31, no. 15, pp. 26212628, 2013.

[3] V. Curri et al, "Time-division hybrid modulation formats: Tx operation strategies and countermeasures to nonlinear propagation," in Proc. Optical Fiber Communication Conf. and Exposition (OFC), paper Tu3A.2, 2014.

[4] M. Qiu et al, "Subcarrier Multiplexing Using DACs for Fiber Nonlinearity Mitigation in Coherent Optical Communication Systems", in Proc. OFC, 2014.

[5] P. Poggiolini et al, "Analytical and Experimental Results on System Maximum Reach Increase Through Symbol Rate Optimization”, J. Lightw. Technol., vol. 34, no. 8, pp. 1872-1875, 2016.

[6] T. Rahman et al, "Digital Subcarrier Multiplexed Hybrid QAM for Data-rate Flexibility and ROADM Filtering Tolerance", in Proc. OFC, paper Tu3K.5, 2016. 